\title{
Gastrointestinal Stromal Tumor with a Novel Mutation of KIT Proto-oncogene
}

\author{
Ryo Fukuda, Naoharu Hamamoto, Yasushi Uchida, Kohichiro Furuta, Tomoko Katsube, Hideaki Kazumori, \\ Shunji Ishinara, Kazutoshi Amano, Kyoichi Adachi, Makoto Watanabe and Yoshikazu Kinoshita
}

\begin{abstract}
The majority of human gastrointestinal mesenchymal tumors are gastrointestinal stromal tumors (GISTs). Recent reports have shown the existence of gain-of-function mutations in the juxta-membrane domain of receptor tyrosine kinase (KIT) in GISTs. We present a 77-year-old woman with GIST diagnosed by positive immunostaining of cluster designation (CD) 34 and KIT. This case had a novel mutation at codon 576 located in the juxta-membrane domain of KIT. Our results indicate the importance of mutations in this KIT region and suggest the possibility of the existence of other types of mutations in this region in GISTs.

(Internal Medicine 40: 301-303, 2001)
\end{abstract}

Key words: CD34, interstitial cells of Cajal, direct sequencing

\section{Introduction}

A large number of interstitial tumors found in the human gastrointestinal tract are designated as gastrointestinal stromal cell tumors (GISTs), since they exhibit typical features different from either smooth muscle cells or Schwann cells (1). Positive immunostaining of cluster designation (CD) 34 and receptor tyrosine kinase (KIT) indicates that most GISTs are derived from interstitial Cajal cells (2-4). KIT, a receptor tyrosine kinase $(5,6)$, and its ligand stem cell factor play a crucial role in the development of interstitial cells of Cajal (7-8). To date, gain-of-function mutations in KIT have been reported in these tumors $(4,9)$. Interestingly, all of these mutations were confined within the KIT juxta-membrane domain. Thus, mutations in this region seem to be associated with the tumorigenesis of GISTs. In the present report, we investigated whether or not any mutation existed in this region in a case of GIST.

\section{Case Report}

A 77-year-old Japanese woman came to us suffering from a gastric tumor. A $6.0 \times 4.5 \mathrm{~cm}$ submucosal tumor was detected at the upper body greater curvature of the stomach. Under the diagnosis of a gastric sarcoma, the tumor was surgically resected.

Morphologically, the tumor was located in the submucosa of the stomach and protruded from the serosa membrane. The section was myxoid and contained a cystic region with a small amount of blood. Histologically, the tumor was comprised of spindle-shaped cells with plump nuclei. Cell density was high and the mitotic index was less than 5 in consecutive 50 highpower fields. The degree of dysplasia was mild to moderate. Immunohistochemically, the tumor was negative for desmin, muscle specific actin, smooth muscle actin, S100, and vimentin staining. However, it was strongly positive for KIT (Fig. 1A) and CD34 staining (Fig. 1B). The genomic sequence of the juxta-membrane region and carboxyl terminus of the KIT protooncogene was determined using DNA extracted from the tumor portion of the paraffin-embedded section and peripheral WBC. These regions were amplified by a nested polymerase chain reaction and subcloned followed by sequencing using a dideoxy chain termination method. No mutation was seen in any codon between 550 to 560 . However, a point mutation (C to $\mathrm{T}$ ) at codon 576 which converts prolin to leucin was detected in 4 of the 8 clones investigated (Fig. 2). No mutation was seen in the DNA from WBC.

\section{Discussion}

In human GISTs, all the gain-of-function mutations were first thought to be confined within the 11 amino acids (codon 550 to codon 560) (4), and later a deletion mutation at codon 578 was reported as a novel gain-of-function mutation (9). Although a mutation in the KIT tyrosine kinase domain has been shown to be another gain-of-function mutation, it has only been seen in the mast cell neoplasm (10-12). These findings indicate that mutations in the KIT juxta-membrane domain has a

From the 2nd Department of Internal Medicine, Shimane Medical University, Izumo

Received for publication January 28, 2000; Accepted for publication August 7, 2000

Reprint requests should be addressed to Dr. Ryo Fukuda, the 2nd Department of Internal Medicine, Shimane Medical University, 89-1, Enya-cho, Izumo, Shimane 693-8501 


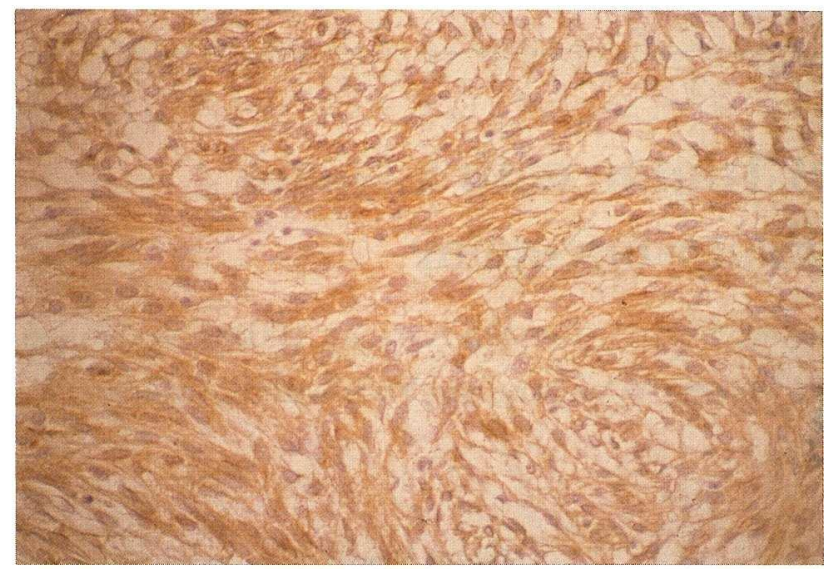

A

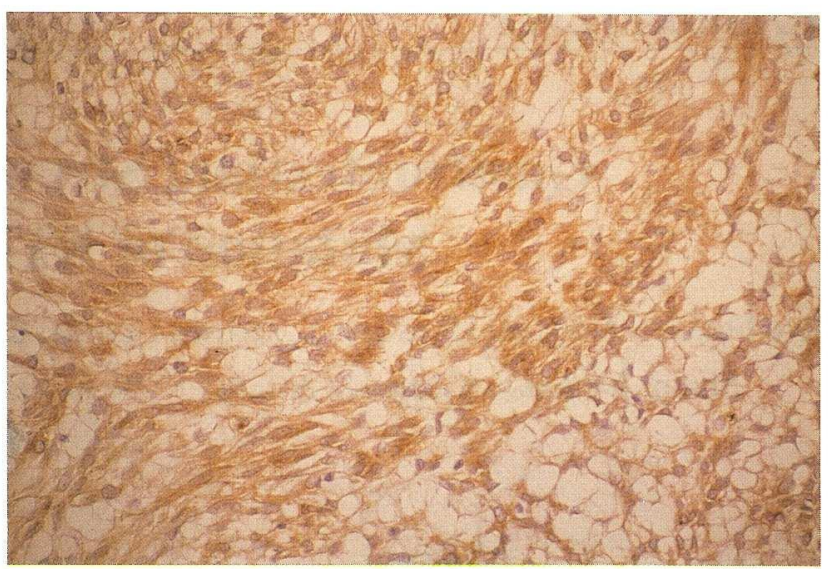

B

Figure 1. Immunostaining for CD34 and KIT. The tumor was positive for both cluster designation (CD) 34 (A) and receptor tyrosine kinase (KIT). (B) Immunoperoxy method $(\times 200)$.

WBC

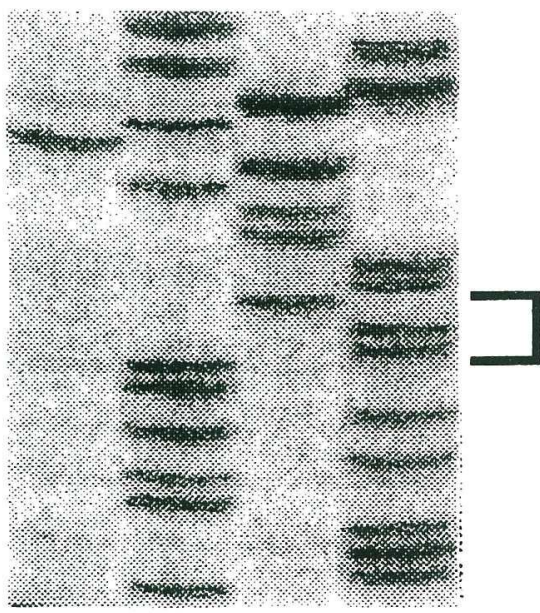

G $\quad \mathrm{A} \quad \mathrm{T} \quad \mathrm{C}$
GIST
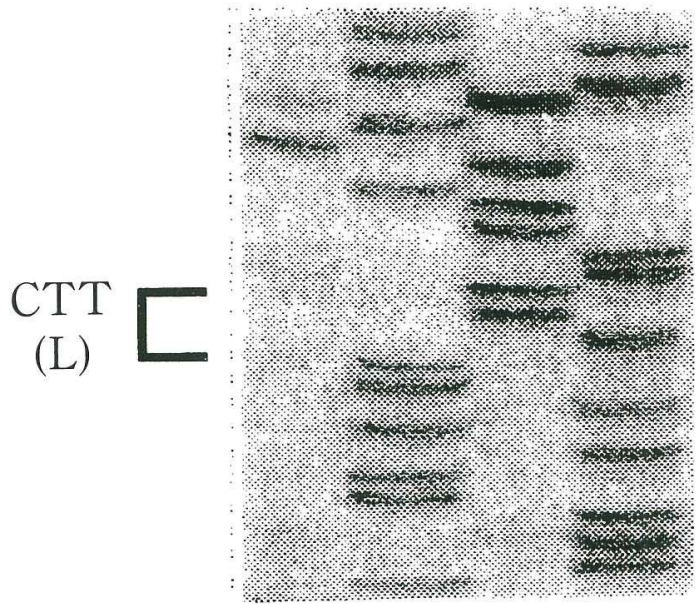

G $\quad A \quad T \quad$ C

Figure 2. Sequence of genomic DNA of the KIT proto-oncogene. A point mutation from C to T was detected at codon 576 in the tumor (GIST). No mutation was seen in DNA from WBC. P: prolin, L: leucin, KIT: receptor tyrosine kinase.

crucial role with GIST tumorigenesis.

The case presented in this report was diagnosed as a GIST from the positive staining of CD34 and KIT. However, no mutation was detected within the 11 amino acids or at codon 578 , which have been reported previously $(4,9)$. Interestingly, we found a novel mutation at codon 576 in the KIT proto-oncogene. This novel mutation was also located in the juxta-membrane domain like other mutations. Whether or not this is a gain-offunction mutation is not clear. However, half of the genomic
DNA clones from the tumor and all the DNA from the WBC had no mutation in this region. Together with the fact that all mutations in human GISTs have been confined within this region, our findings suggest the possibility that this point mutation is also a KIT gain-of-function mutation. Recently, a germline mutation of the KIT proto-oncogene has been shown in some of the members of familial GISTs by detection of KIT mutation in DNA from WBC (13). However, the present case had no such family history of gastrointestinal tumors nor the 


\section{GIST with a Novel Mutation in KIT}

mutation in genomic DNA from WBC.

Although further investigation is necessary to reconcile whether this novel mutation is a gain-of-function mutation or not, our results indicate the importance of mutations in this KIT region and suggest the possibility of the existence of other types of mutations in this region in GISTs.

\section{References}

1) Fisherman AP. Gastrointestinal tract. in: Ackerman's Surgical Pathology. Rosai J, Ed. 8th ed. St. Louis, MO, Mosby, 1996: 645-653.

2) van de Rijn M, Hendrickson MR, Rouse RV. CD34 expression by gastrointestinal tract stromal tumors. Hum Pathol 25: 766-771,1994.

3) Miettinen M, Virolainen M, Maarit-Sarlomo-Rikala M. Gastrointestinal stromal tumors value of CD34 antigen in their identification and separation from true leiomyomas and schwannomas. Am J Surg Pathol 19: 207216, 1995.

4) Hirota S, Isozaki K, Moriyama $Y$, et al. Gain-of-function mutations of $c$ kit in human gastrointestinal stromal tumors. Science 279: 577-580, 1998.

5) Besmer P, Murphy JE, George PC, et al. A new acute transforming feline retrovirus and relationship of its oncogene $v$-kit with the protein kinase gene family. Nature 320: 415-421, 1986.
6) Chabot B, Stephenson DA, Besmer P, Bernstein A. The proto-oncogene $c$-kit encoding a transmembrane tyrosine kinase receptor maps to the mouse. Nature 335: 88-89, 1988.

7) Williams DE, Eisenman J, Baird A, et al. Identification of a ligand for the c-kit proto-oncogene. Cell 63: 167-174, 1990.

8) Huizinga JD, Thuneberg L, Kluppel M, Malysz J, Mikkelsen HB, Bernstein A. W/kit gene required for interstitial cells of Cajal and for intestinal pacemaker activity. Nature 373: 347-349, 1995.

9) Nakahara M, Isozaki K, Hirota S, et al. A novel gain-of-function mutation of $c$-kit gene in gastrointestinal stromal tumors. Gastroenterology 115: 1090-1095, 1998.

10) Furitsu $T$, Tsujimura $T$, Tono $T$, et al. Identification of mutations in the coding sequence of the proto-oncogene $c$-kit receptor tyrosin kinase in a human mast cell leukemia cell line causing ligand-independent activation of $c$-kit product. J Clin Invest 92: 1736-1744, 1993.

11) Tujimura $T$, Furitsu $T$, Morimoto $M$, et al. Substitution of an aspartic acid results in constitutive activation of $c$-kit receptor tyrosine kinase in a rat tumor cell line RBL-2H3. Int Arch Allergy Immunol 106: 377-385, 1995.

12) Tsujimura $T$, Furitsu $T$, Morimoto $M$, et al. Ligand-independent activation of $c$-kit receptor tyrosine kinase in a murin mastocytoma cell line $\mathrm{p}$ 815 generated by a point mutation. Blood 83: 2619-2626, 1994.

13) Nishida $T$, Hirota $S$, Taniguchi $M$, et al. Familial gastrointestinal stromal tumors with germline mutation of the KIT gene. Nat Genet 19: 323-324, 1998 (letter). 